\title{
1 Interaction of a Biosurfactant, Surfactin with a Cationic \\ 2 Gemini Surfactant in Aqueous Solution
}

3 Lei Jin, ${ }^{\dagger, 1}$ Vasil M. Garamus, ${ }^{\dagger, 2}$ Fang Liu, ${ }^{1}$ JingwenXiao, ${ }^{1}$ HelmutEckerlebe ${ }^{2}$,

4 RegineWillumeit-Römer, ${ }^{2}$ BozhongMu, ${ }^{1}$ andAihuaZou ${ }^{*}, 1$

5

${ }^{1}$ Shanghai Key Laboratory of Functional Materials Chemistry, State Key

$24{ }^{*}$ To whom correspondence should be addressed.

25 Tel/Fax.: +86-21-64252231.

26 E-mail: aihuazou@ecust.edu.cn.

$27 \quad{ }^{1}$ East China University of Science and Technology.

$28{ }^{2}$ Helmholtz-ZentrumGeesthacht.

$29{ }^{\dagger}$ These authors contributed equally to this work. 
30 Abstract:The interaction between biosurfactantSurfactin and cationic Gemini 4 surfactant ethanediyl-1,3-bis(dodecyldimethyammoniumbromide) (abbreviated as 12-3-12) was investigated using turbidity, surface tension, dynamic light scattering(DLS)andsmallangle neutron scattering (SANS). Analysis of critical micelle concentration (CMC)values in Surfactin/12-3-12 mixture indicatesthat there issynergismin formation of mixed Surfactin/12-3-12 micelles.Although Surfactin and 12-3-12 are oppositelycharged inphosphate buffer solution (PBS, $\mathrm{pH} 7.4$ ), there are no precipitates observedat the concentrations below theCMCofSurfactin/12-3-12 system. However, at the concentration above CMC value, the Surfactin/12-3-12 mixture is severely turbid with high 12-3-12 content. DLS and SANS measurements follow thesize and shapechanges of mixed Surfactin/12-3-12 aggregates from small spherical micelles via elongated aggregates to large bulk complexes with increasing fraction of Gemini surfactant.



5

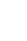

48
1 2 3

(1)

Keywords: Surfactin, Gemini surfactant, micelle, surface tension, SANS 57 58 


\section{Introduction}

The mixtures of different types of surfactants have attracted significant interest sincemixtures provide a synergistic enhancement of performance and functionality which cannot take place in single surfactant systems ${ }^{1-3}$. Synergism increases with thedegree of charge difference ${ }^{4-6}$ meaning that the higher level of synergism is obtained by mixing anionicand cationic surfactants. There have been plenty of studies of anionic/cationic surfactantsmixtures. The behavior and physicochemical properties of mixturesof a highly branched cationic and sodium alkyl sulfatehave been widely studied by $\mathrm{Yu}$ and co-workers ${ }^{7-9}$. And, it has been reported that the asymmetrically double-tailed cationic surfactantsfail to fit well into a crystal lattice structure and hence minimize precipitation ${ }^{10}$.Bergström and co-workershave studied the structures in cationic/anionic surfactant including Geminisystems using thesmallangle neutron scattering technique ${ }^{11-13}$. However, precipitation is a common phenomenonthat can occur in all of the anionic/cationic surfactants mixturementioned aboveand in most casesprecipitation is undesirable because it rendersthe surfactant ineffective in solution. Therefore, a better understandingof such system is necessary to broaden the horizon for their application.

Surfactin, a biosurfactant produced by various Bacillus subtilis strains, is cyclic lipopeptide built from a heptapeptide (Glu-Leu-D-Leu-Val-Asp-D-Leu-Leu) and a $\beta$ hydroxy fatty acid with variable chain lengths of 13-15carbon atoms ${ }^{14-16}$ (Figure 1(a)).Surfactin has been receiving attention for industrial,biotechnological, and therapeutical applications ${ }^{17,18}$ because of its high surface activity, which could reduce the surface tension of water to $27 \mathrm{mN} / \mathrm{m}^{17}$ by that concentration as low as $10^{-5} \mathrm{M}$. It can be seen that Surfactinhas two $-\mathrm{COOH}$ groups in L-Glu1 and L-Asp5. The $\mathrm{pK}_{\mathrm{a}}$ values $^{19}$ of Asp and Gluarearound 4.3 and 4.5, respectively, which means it exists as an anionic molecule in neutral solution.

Gemini surfactants contain two single-chain surfactant moietiesjoined by a spacer group. ${ }^{20-22}$ The two amphiphilic moieties are close to each other, especially when the spacer group is short. Owing to this, on the one hand, the interaction between the hydrophobic chains is enhanced; on the other hand, repulsion between the hydrophilic 
groups (particularly the electrostatic between the ionic head groups) will be greatly reduced due to the chemical bonds connection. This novel class of surfactants hasmany unique properties that are superior to those of conventionalsingle-chain surfactants, such as remarkably low critical micelleconcentration (CMC), much higher surface activity, and better wetting properties. Moreover, it has unusualaggregation morphologies. Since the surfactant micellization is driven by hydrophobic interactionsbut opposed by the repulsion of charged head groups(for ionic surfactants) and hydration (for nonionic surfactants), Gemini surfactant is more readily to form aggregates. The quaternary ammonium Geminisurfactantsare widely investigated about their properties andapplications ${ }^{23,24}$. For the 12-s-12 (12 carbon atoms on the hydrophobic alkyl chain and 's' represents the number of carbon atoms of the spacer) series, at the concentration close to $\mathrm{CMC}$ value, spherical micelles are formed. Increasing the surfactant concentration, for the short spacer, such as $\mathrm{s}=2,3$, elongated rod-like micelles are formed; for the medium length like $\mathrm{s}=4,6,8,10,12$, spherical micelles are formed, and for the longer spacer, $s=16,20$, vesicles are readily to form ${ }^{24}$.

There are a lot of studiesonSurfactin ${ }^{14-19}$ and Gemini ${ }^{20-24}$ from various aspects. However, to the best of ourknowledge, there is no research about their mixtures.The dynamic interfacial tension between the Surfactin/12-3-12binary aqueous system and crude oil has been carried out in our group, which revealed that Surfactin/12-3-12 mixtures can reduce the interfacial tensionto an ultralow level in alkaline environment(seedetails in Supporting Information) which can be used in the application inoil recovery. The better understanding of the fundamental physicochemical property of the mixture of Surfactin/Gemini surfactant system is the key point for their practical application.In our previous work, we have investigated the micelle formationand interfacial behavior of Surfactin ${ }^{25}$ in PBS buffer.A further research ${ }^{26}$ has also been carried out on the interaction of Surfactin with betaines which revealed that there was a synergistic effect between them, and the configuration of mixed micelles changed with the head-groups of betaines.Then, the interactions among neutral polymer PAM,Surfactin and four betainesin PBSbuffer were 
furtherstudied. Transition from spherical to rodlikeaggregates (micelles) has been

observed

in

solutions

of

Surfactin

and

122 Surfactin/SDDAB(N-dodecyl-N,N-dimethyl-3-ammonio-1-propanesulfonate)with addition of $0.8 \mathrm{wt} \%$ of $\mathrm{PAM}^{27}$. Wang et $\mathrm{al}^{28}$ studied the interaction between surfactin and cationicsurfactant CetyltrimethylammoniumBromide(CTAB) in mixed micelle, There exited synergism between anionic Surfactincationic surfactantCTAB, and the mixed system might be ableto form vesicle spontaneously at high molar fraction ofsurfactin.However they did not study the precipitationcondition and the aggregates properties(shape and size)of the anionic/cationic mixed surfactant aqueous system. A continuation of the aggregation behavior of Surfactin/12-3-12 mixtures were investigatedin presentpaper by surface tension, smallangle neutron scattering and dynamic light scattering, which will broaden thepotential application forboth Surfactin andGemini surfactants.

\section{Materials and Experiments}

\subsection{Materials.}

2.1.1 Surfactin. Surfactin was produced by Bacillus subtilis TD7 cultured in a laboratory of East China University of Science and Technology ${ }^{29,30}$.Surfactin isoform (Figure 1(a)) was separated by extraction with anhydrous ether, isolated with normal pressure ODS C18 column and purified by the RP-HPLC (Jasco, Japan). The structure of the isolated lipopeptide was determined by the electrospray ionization-time-of-flight mass spectrometer (ESI-TOF MS/MS) and GC/MS ${ }^{29,30}$. 


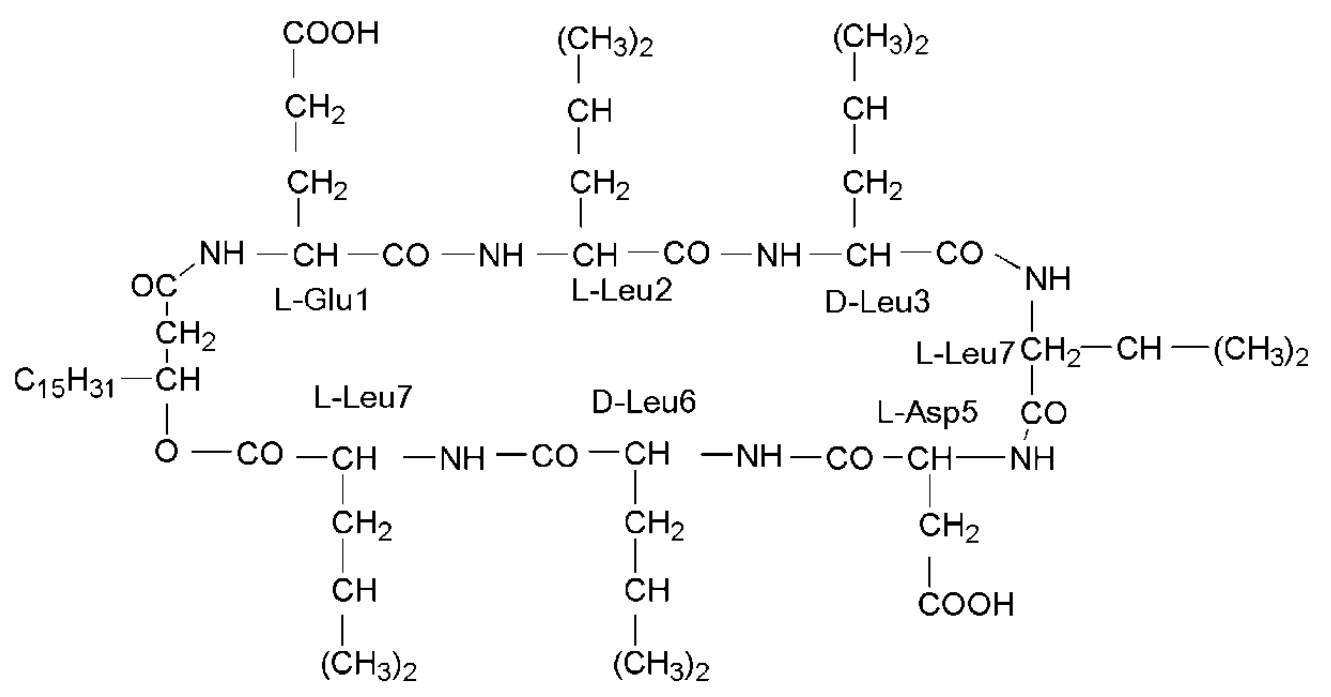

a

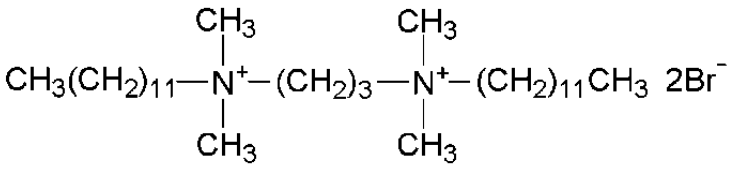

Figure 1.The chemical structure of Surfactin(a) and 12-3-12(b).

2.1.2 GeminiSurfactant. Crude ethanediyl-1,3-bis(dodecyldimethylammonium) bromide (abbreviated as 12-3-12) was obtained fromDaochun Chemical Technology Co. Ltd. It was recrystallized at least four times with ethanol and ethyl acetate ${ }^{31}$ then dried under vacuum with low heat at $40^{\circ} \mathrm{C}$ for 1 day.The purity of $12-3-12$ was

147 checked by ${ }^{1} \mathrm{H}$ NMR.The ${ }^{1} \mathrm{H}$ NMR Spectra of 12-3-12 and the relatedanalyzingdataarepresented in Supporting Information, and the impurity content was estimated to be below $2 \%$.The chemical structure of $12-3-12$ is presented in Figure1(b).

2.2 Sample Preparation. First, $10 \mathrm{mM}$ phosphate buffer solution (PBS, $\mathrm{pH}=7.4$,thispH value was chosento achieve better water solubility forSurfactin) were prepared as the solvent, then high concentration of pure surfactant solutions were prepared by weighing the desired amounts of surfactants and dissolved in the solvent. The mixtures of the desired composition of surfactants were made by mixing high concentration of two pure surfactant solutions with a certain amount and added 
solvent to a settled volume. The composition of the Surfactin/12-3-12 binary system is described with $\alpha_{\text {Surfactin, }}$ the mole fraction of Surfactin.

$$
\alpha_{\text {surfactin }}=\frac{c_{\text {surfactin }}}{c_{\text {surfactin }}+C_{12-3-12}}
$$

For SANS experiments, the samples were prepared under the same condition except doubly distilled water was replaced by $\mathrm{D}_{2} \mathrm{O}(99.9 \% \mathrm{D}$, from Sigma-Aldrich).The $\mathrm{pD}$ value was mediated by eq2, with proper corrections by Krezel et $\mathrm{al}^{32,33}$.

$$
\mathrm{pD}=0.929 \mathrm{pH} \text { meter trading }+0.42
$$

2.3 Precipitation Determination.For the series of mixed solutions of Surfactin and 12-3-12, since surfactant solutions can remain supersaturatedfor long periods of time before precipitation started ${ }^{34,35}$, therefore, to determine whether there is precipitationformed, all solutionswere cooled at $0^{\circ} \mathrm{C}$ to force precipitation to $\operatorname{occur}^{35}$.The solutions were then placed in a water bath at $25.0 \pm 0.1{ }^{\circ} \mathrm{C}$, shaken periodically, and allowedto equilibrate for at least 4 days beforemeasurements. If a solution isoutside of the precipitation region, crystalswould dissolve so that the solution becameisotropic. If crystals remained in solution afterequilibration, the initial solution compositionwas considered to be inside the precipitationregion. The presence of precipitate in solution wasdetermined by visual inspection using a high intensity light.

2.4Surface Tension Measurements.For CMC measurements, surface tension was measuredafter the samples were proper mixed by ultra-sonic and equilibratedfor 1 day. Measurements were carried out at $25^{\circ} \mathrm{C}$ by surface tension meter DCAT2 1 (Dataphysics, Germany) using a Wilhelmy small platinum plate of ca. $4 \mathrm{~cm}$ perimeter. The plate was first rinsed with doubly distilled water and then burned to redto make surethere are no surfactants left on the plate.Prior to the measurements, theequipment was tested by determination of a surface tension ofdouble distilled water(72 \pm 0.2 $\mathrm{mN} / \mathrm{m})$ at $25.0^{\circ} \mathrm{C}$.

2.5DLSMeasurements. DLS measurements werecarried out at $25^{\circ} \mathrm{C}$ with Nano-ZS (Malvern Instruments Ltd. UK), equipped with $4 \mathrm{~mW}$ He-Ne laser at $\lambda_{0}=633 \mathrm{~nm}$ as a light source. PAL (Phase Analysis Light Scattering) and LDV (Laser Doppler 
Velocimetry) were used to measure the size at a scattering angle of $173^{\circ}$. Samples were kept in quartz cuvette. DLS measurements were made at $0.5 \mathrm{mM}$ surfactant concentration with $\alpha_{\text {Surfactin }}$ ranges from 0 to 1 , and each sample was measured for three times.Experimental data aregivenby the Malvern Zetasizer software.

2.6Small angle neutron scattering (SANS) Measurements.SANS measurements were performed at SANS1 instrument operated by HZG and TUMat the Meier-Leibniz-Zentrum (FRM2, Garching, Germany) ${ }^{36}$. The wavelength of the neutron beamwas $6 \AA$ with $\Delta \lambda / \lambda=10 \%$. A position-sensitive detector with $128 \times 128$ pixels and a pixel size of $8 \mathrm{~mm}$ was used. These settings allowed us to cover a $q$ range from 0.008 to $0.4 \AA^{-1}$.Samples were placed in $1 \mathrm{~mm}$ thick quartz cellandmeasured atT $=25 \pm 1{ }^{\circ} \mathrm{C}$. Data have been normalized by water measurements at same geometry and corrected for detector response and electronic noise. The scattering from PBS buffer prepared in $\mathrm{D}_{2} \mathrm{O}$ wassubtracted as the background. Data reduction has been done by the QtiKWS ${ }^{37}$ data analysissoftware.The dispersion of resolution function has been calculated and taken into account for further data analysis.

\section{Results and Discussion}

3.1Precipitation Phase Boundary.SinceSurfactin and 12-3-12 are oppositely charged in PBS (pH7.4), it wasexpected that precipitates will form in their mixtures.In order to evaluate the precipitation potential of these mixtures as well as to ensure that the surface tension studies were conducted in regions without precipitates, turbidity studies were conducted.At a constant $\alpha_{\text {Surfactin }}$ value,themeasurements were made at seriesconcentrations to confirmthe phase boundaries.

In Figure 2(a), the red line representsthe precipitation phase boundary which was determined by visual observationusing a high intensity light. The precipitation region was further divided into two parts as dark gray and light grey in Figure 2(a), where the precipitation potential is different between these two parts. In order to better explain the meaning of "dark and light grey" in Figure 2(a), the photos of 0.5

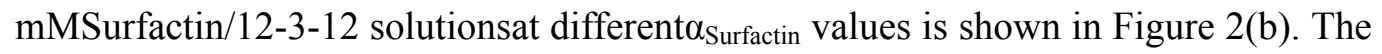
dark gray represents high turbid solutions $\left(\alpha_{\text {Surfactin }}=0.1,0.2\right.$, and 0.3 in Figure 2(b)) which are entirely opaque, while the light gray corresponds to turbid solutions 
$\left(\alpha_{\text {Surfactin }}=0.4,0.5\right.$, and pureGemini in Figure 2(b))which can still transfer light. Besides, the white region means visually clear and transparent solutions ( $\alpha_{\text {Surfactin }}$ $=0.6-1$ in Figure 2(b)).
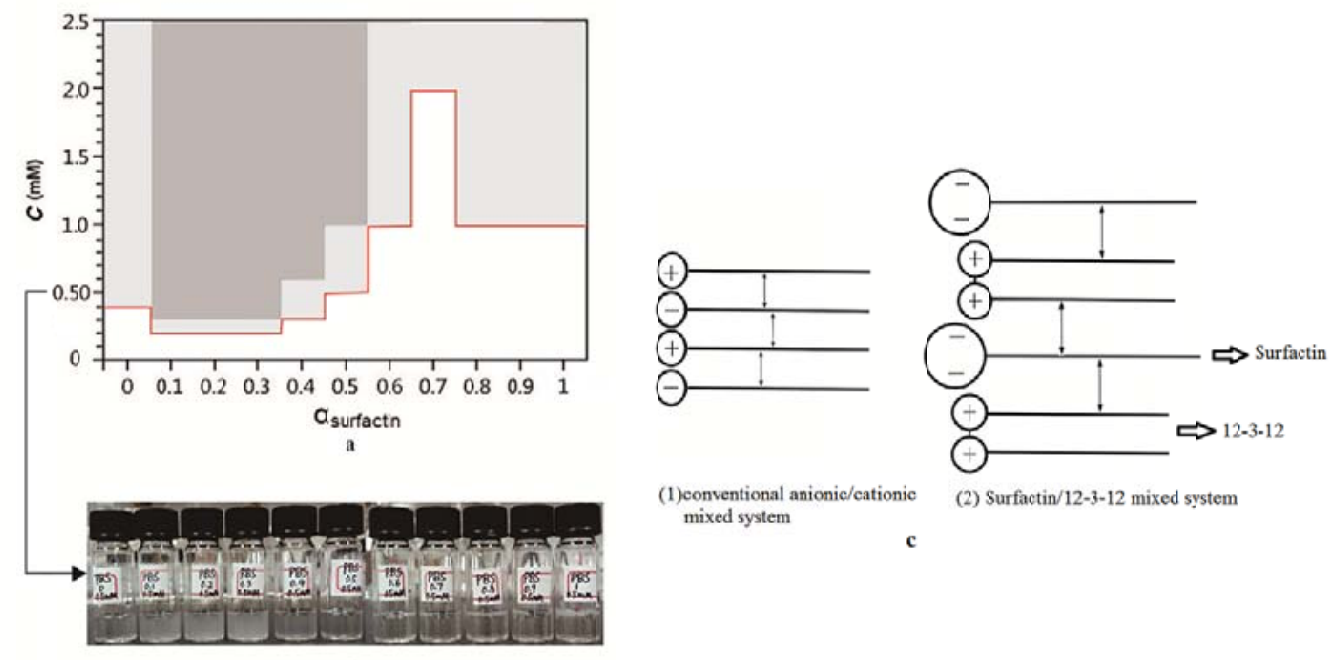

Figure 2.a:Theprecipitation phase boundary ofSurfactin/12-3-12mixtureover a wide range of concentration,b:The photos of0.5mMSurfactin/12-3-12solutions with $\alpha_{\text {Surfactin }}$ ranges from 0 to 1,c:The peptide ring of Surfactincan result inreducedprecipitation, $\mathrm{c}(1)$ is the precipitationschematic diagram of conventional anionic/cationic mixtures (Adapted fromPanswad ${ }^{35}$ ).

It was reportedthat pure 12-3-12aqueous solution of concentration close to $1 \mathrm{wt} \%$ $(16 \mathrm{mM})$ was still clear by visual observation at $25^{\circ} \mathrm{C}^{38}$. However,it can be seen from Figure 2(a) that the $0.4 \mathrm{mM} 12-3-12$ solution is turbid when it was dissolved in $10 \mathrm{mM}$ PBS buffer.This result means that there are big aggregates formed in 12-3-12 solution. Therefore, PBS buffer should have someeffect on the aggregation behavior of 12-3-12. Thenegatively chargedhydrogen phosphates and dihydrogen phosphates will screen the positive charges on the head groups of 12-3-12 and weaken the electrostatic repulsion between 12-3-12 molecules, thuslarge aggregates of 12-3-12formedin PBS buffer.Figure 2(a) also shows thatthe solution in the high 12-3-12 content region is more turbid and the regionof dark grey is much larger than that in the high Surfactin content region. In aqueous solution the peptide ring of Surfactin shows a "horse-saddle" topology ${ }^{39}$, the two negatively charged amino acid residues Glu and 
Asp constitute a minor polar domain. On the opposite side, Val residue extends down, facing the fatty acid chain, making up a major hydrophobic domain. When mixed with 12-3-12,the hydrophobic domain of Surfactinsterically hindered the interaction between oppositely charged head groups and tail-tailinteraction (see in Figure 2(c)). In Panswad'swork ${ }^{35}$,extended anionicsurfactants(a novel class of surfactants thathave groups of intermediate polarity such as polypropyleneoxides (PO) or ethylene oxides (EO) inserted between thehydrocarbon tail and the hydrophilic head group) have the similarproperty whenmixed with cationic surfactants.

So there are no precipitates formed in Surfactin/12-3-12 mixture at low surfactant concentration. The CMCvalues of Surfactin/12-3-12 mixture presented in Table 1with different $\alpha_{\text {Surfactinvalues, which were determined by surface tension measurements as }}$ shown in Figure 3. It can be found that all CMC values are smaller than the surfactant concentrations suggested bythe precipitation phase boundary in Figure 2(a). And it is interesting that the precipitates region reduced with theincrease of $\alpha_{\text {Surfactin }}$ value.This excellent property can mitigate the main disadvantage of mixed anionic and cationic surfactant systems which is their tendency to form precipitates ${ }^{40}$. The precipitation happening in the anionic/cationic surfactant system is unfavorable fortheir applications, such as detergency performance and subsurface remediation of oil contamination $^{40,41}$.

3.2 Interaction betweenSurfactin and Gemini (12-3-12). Since the precipitationphase boundary of Surfactin/12-3-12 mixturehas been studied, the synergism between them was expected and further studied. The surface tension $(\gamma)$ as afunction of total surfactant concentration with different molar fractions of Surfactin is presented in Figure3. The relevant results obtained from the analysis of surface tension data are summarized in Table 1. 


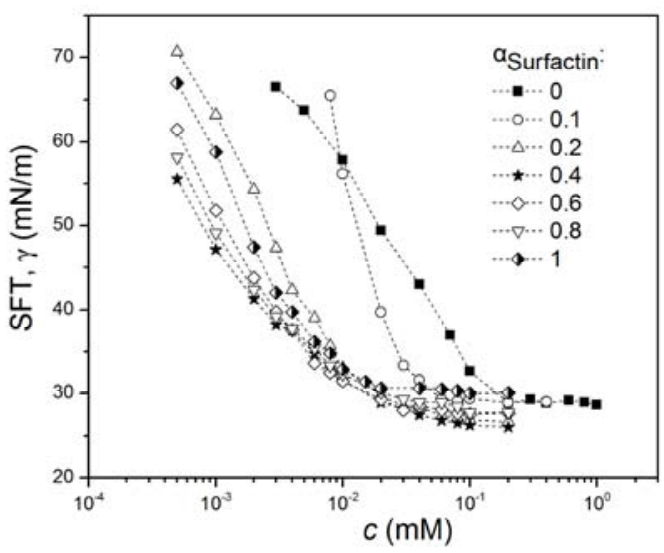

261

262 Figure 3.Surface tension isotherm of Surfactin/12-3-12 system in $10 \mathrm{mM}$ PBS

263 medium at $25^{\circ} \mathrm{C}$.

264 
Table 1.CMCand $\gamma_{C M C}$ Values of Surfactin/12-3-12 system

\begin{tabular}{cccc}
\hline$\alpha_{\text {Surfactin }}$ & $\begin{array}{c}\mathbf{C M C}_{\text {exp }} \times \mathbf{1 0}^{\mathbf{2}} \\
(\mathbf{m M})\end{array}$ & $\begin{array}{c}\mathbf{C M C}_{\text {ideal }} \times \mathbf{1 0}^{\mathbf{2}} \\
\mathbf{( m M )}\end{array}$ & $\begin{array}{c}\gamma_{\mathbf{C M C}}(\mathbf{m} \mathbf{N} / \mathbf{m}) \\
( \pm 0.03)\end{array}$ \\
\hline 0 & $18.9 \pm 0.3$ & $18.9 \pm 0.3$ & 30.43 \\
0.1 & $3.75 \pm 0.01$ & $6.91 \pm 0.02$ & 30.39 \\
0.2 & $1.21 \pm 0.02$ & $4.23 \pm 0.03$ & 30.15 \\
0.4 & $1.14 \pm 0.01$ & $2.38 \pm 0.02$ & 29.77 \\
0.6 & $1.05 \pm 0.03$ & $1.66 \pm 0.05$ & 28.91 \\
0.8 & $1.06 \pm 0.01$ & $1.27 \pm 0.03$ & 30.32 \\
1 & $1.03 \pm 0.03$ & $1.03 \pm 0.03$ & 30.97 \\
\hline
\end{tabular}

266

Figure 3 presents the surface tension results of pure Surfactin, 12-3-12, and their mixtures at $\alpha_{\text {Surfactin }}=0.1,0.2,0.4,0.6$ and 0.8 . Compared with that of pure Surfactinand 12-3-12, the $\gamma_{\mathrm{CMC}}$ values of Surfactin/12-3-12 mixtures are smaller, all also in the surface tension decreasing region before $\mathrm{CMC}$, a lower surfactants concentration is needed to reach the same surface tension.

Table 1 shows that the CMC of $12-3-12$ is $1.89 \times 10^{-1} \mathrm{mMin} 10 \mathrm{mM}$ PBS $(\mathrm{pH}=7.4)$. Shangetal ${ }^{42}$ hasreported that the $\mathrm{CMC}$ of $12-3-12$ in aqueous solution is 9.21 $\times 10^{-1} \mathrm{mM}$, while its $\mathrm{CMC}$ decreased to $1.16 \times 10^{-1} \mathrm{mM}$ when $5 \mathrm{mMNaBr}$ was added in the system,which is close to our result.The surface activity of 12-3-12 changes

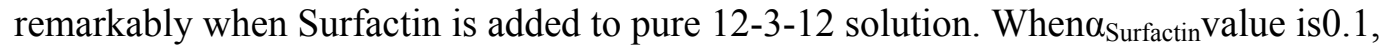
The $\mathrm{CMC}$ value is reduced to $3.75 \times 10^{-2} \mathrm{mM}$ (Table 1 ) by one order of magnitude compared with that of pure 12-3-12. With $\alpha_{\text {Surfactinincreasesto }} 0.8$, theCMCvalue gradually decreases andapproachesto that of pure Surfactin. The lower $\gamma_{\mathrm{CMC}}$ and CMC values show that the mixture of Surfactin and 12-3-12 has better ability and efficiency in reducing surface tension than the individual surfactant.

The regular solution theory $(\mathrm{RST})$ is applied to obtainCMC $\mathrm{ideal}_{\mathrm{V}}$ alues for the mixed

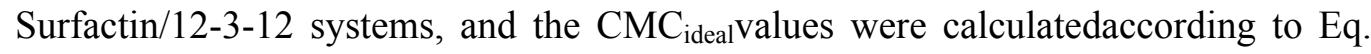
$3^{43}$.Here 1 and 2 refers to Surfactin and 12-3-12, respectively. Parameters $\alpha_{1}$ is the molar fraction of Surfactin, $f_{1}$ and $f_{2}$ are the activity coefficients of Surfactin and 
12-3-12 in mixed micelles (for ideal mixing $f_{1}=f_{2}=1$ ), respectively.

$$
\frac{1}{\mathrm{CMC}_{\text {ideal }}}=\frac{\alpha_{1}}{f_{1} \mathrm{CMC}_{1}}+\frac{\left(1-\alpha_{1}\right)}{f_{2} \mathrm{CMC}_{2}}
$$

286

287

288

The experimental CMC values (Table 2) are always lower than the values expected for ideal mixing, indicating that there is synergism pointing to the formation of mixed micelles inSurfactin/12-3-12solution.

The strong synergistic behavior is also supported by negative values of Molecular Interaction Parameter $\beta^{\mathrm{m}}$ calculatedwith Eq. 4 and 5 according to the Regular Solution Theory ${ }^{44}$.

$$
\begin{gathered}
\frac{\left(X_{1}^{\mathrm{m}}\right)^{2} \ln \left[\left(\alpha_{1} C_{12}^{\mathrm{m}} / X_{1}^{\mathrm{m}} C_{1}^{\mathrm{m}}\right)\right]}{\left(1-X_{1}^{\mathrm{m}}\right)^{2} \ln \left[\left(1-\alpha_{1}\right) C_{12}^{\mathrm{m}} /\left(1-X_{1}^{\mathrm{m}}\right) C_{2}^{\mathrm{m}}\right]}=1 \\
\beta^{\mathrm{m}}=\frac{\ln \left(\alpha_{1} C_{12}^{\mathrm{m}} / X_{1}^{\mathrm{m}} C_{1}^{\mathrm{m}}\right)}{\left(1-X_{1}^{\mathrm{m}}\right)^{2}}
\end{gathered}
$$

Where $\alpha_{1}$ is the mole fraction of Surfactin in solution, $X_{1}^{\mathrm{m}}$ is the mole fraction of Surfactin in the mixed micelle. $C_{1}^{m}, C_{2}^{m}$ and $C_{12}^{m}$ is the CMC values of pure Surfactin, pure 12-3-12 and mixturerespectively. The conditions for synergism to exist in the mixture are as follows: (a) $\beta^{m}$ is negative; (b) $\left|\beta^{m}\right|>\left|\ln \left(C_{1}^{m} / C_{2}^{m}\right)\right|$. In Table 2, the values of $\beta^{m}, \quad$ and $\left|\ln \left(C_{1}^{m} \quad / \quad C_{2}^{m}\right)\right|$ obtained for different compositions of Surfactin/12-3-12systemare summarized. It can be seen that the average negative values of the interaction parameter $\beta_{\text {ave }}$ forSurfactin/12-3-12 mixtures support the synergism between Surfactin and 12-3-12.

For conventional anionic/cationic mixtures, the $\beta^{\mathrm{m}}$ values are reported to be in the range of -10 to -20 by Rosen ${ }^{45}$, while the values report here are in the range of -2 to -6.Although Surfactin and Gemini mixtures show lower synergism than conventional surfactant mixtures, this unique combination actually has better ability in practical application due to the less precipitation potential (see Fig. 2(a)and discussion). Wang ${ }^{28}$ and co-workers have investigatedthe synergism of Surfactin/CTAB mixtures using the surface tension methodover a wide range of mole fraction which means the precipitation is not heavy enough to be obstacle to CMC measurement, the $\beta^{\mathrm{m}}$ values of the mixturesare in the range of -1 to -3 which also show much lower 
synergism than conventional anionic/cationic mixtures. Therefore, we canspeculate that Surfactin can reduce precipitation when mixed with not only 12-3-12 but also other kinds of cationicsurfactants due to itsspecial structure.

Table 2.Molecular Interaction and Synergism Parameters for Surfactin/12-3-12 mixtures.

\begin{tabular}{cccc}
\hline$\alpha_{1}$ & $\beta^{\mathrm{m}}$ & $X_{1}{ }^{\mathrm{m}}$ & $\left|\ln \left({C_{1}}^{\mathrm{m}} / C_{2}{ }^{\mathrm{m}}\right)\right|$ \\
\hline 0.1 & -2.68 & 0.5795 & \\
0.2 & -5.77 & 0.5982 & \\
0.4 & -4.54 & 0.6885 & 2.91 \\
0.6 & -4.04 & 0.7654 & \\
0.8 & -3.28 & 0.8695 & \\
$\beta_{\text {ave }}=-4.06$ & & & \\
\hline
\end{tabular}

Prof. Maeda ${ }^{46}$ suggested a new approach which is based on the phase separation model and describes the thermodynamic stability of mixed micelle using Gibbs energy of micellization. The values of $\Delta G_{\mathrm{M}}, B_{0}, B_{1}$, and $B_{2}$ were also calculated and discussed (presented in Support Information).Negative $B_{1}$ values indicate that with an increasing molar fraction of Surfactin, the chain-chain interaction initially gets stronger then becomes weak. The $\Delta G_{\mathrm{M}}$ values also reveal that mixed micelles are more stable than pure surfactant micelles.

\subsection{Structure Study of the Aggregates in Bulk Phase}

3.3.1Dynamic Light Scattering (DLS). DLS was performed to determine the size distribution of the aggregates, as shown in Figure 4 by the volume size distribution.Measurements were made at a constant total surfactantconcentration of 0.5 mM.For pure 12-3-12, it can be seen that large aggregates around 200 nmare mainly formed in PBS, which is consistent with the turbidityshown in figure 2(a). For Surfactin/12-3-12 mixtures, at higher 12-3-12 content region $\left(\alpha_{\text {Surfactin }}=0.1,0.2\right.$, and 0.3), large size aggregateswith hydrodynamic $\mathrm{r}$ around $400 \mathrm{~nm}$ are mainly formed in Surfactin/12-3-12 solution. That is why the Surfactin/12-3-12 solutions at this composition are obviouslyturbid. With the increasing amount of Surfactin $\left(\alpha_{\text {Surfactin }}=\right.$ 
0.4 and 0.5), the size distribution of aggregates also appears thebimodal distribution,most aggregates are around $30 \mathrm{~nm}$, and there remains someamount of large aggregates around $400 \mathrm{~nm}$. Thus, the Surfactin/12-3-12 solution $\left(\alpha_{\text {Surfactin }}=0.4\right.$ and 0.5 )isclear and transparent. When $\alpha_{\text {Surfactin }}$ is 0.6 , there are only small micelles around $6 \mathrm{~nm}$ in Surfactin/12-3-12 solution.At higher Surfactin content region


to about $3 \mathrm{~nm}$. For pure Surfactinsolution, micelles around $4 \mathrm{~nm}$ are mainly formed, which is similarto the result reported by $\mathrm{Zou}^{25}$.
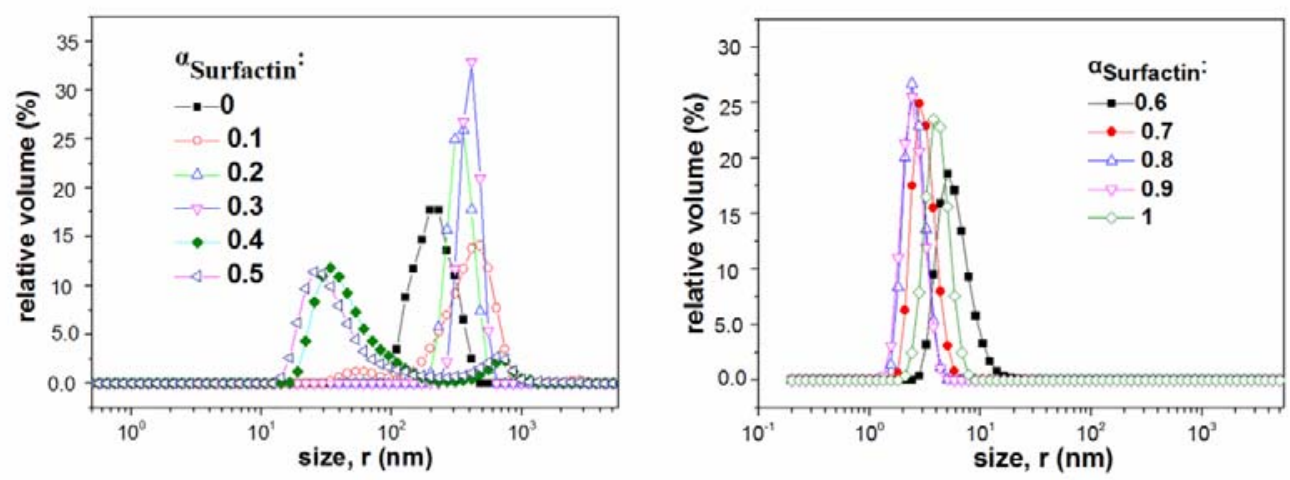

Figure 4. Volume Size Distribution ofSurfactin/12-3-12Aggregates at different $\alpha_{\text {SurfactinValues. }}$

The DLS results indicate that large aggregates are formed in Surfactin/12-3-12 solution at high 12-3-12 content, and the smaller micelles start to form at high Surfactin content. This result agrees well with the phenomenon observed inFigure 2(a), where the severely turbid solution shade into clear and transparent ones with the increasing molar fraction of Surfactin.

\subsubsection{Small Angle Neutron Scattering (SANS) measurements.DLS measures the} hydrated size of particles(particles plus water), whereas SANS points to the "dry size". ${ }^{47}$ SoSANS measurements were performed to further confirm the size and shape changes of Surfactin/12-3-12 aggregates with $\alpha_{\text {Surfactinvalues. Here the measurements }}$ were made at a constant total surfactant concentration of $0.5 \mathrm{mM}$. As shown in Figure 2 , the Surfactin/12-3-12 solution is turbid when $\alpha_{\text {Surfactin }}$ is in the range of0to 0.5 . Therefore, the SANS measurements wereonly performed for pure Surfactin and 


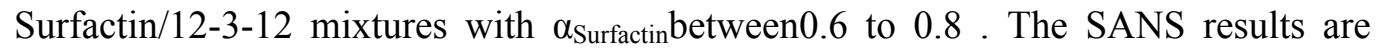
presented in Figure 5.

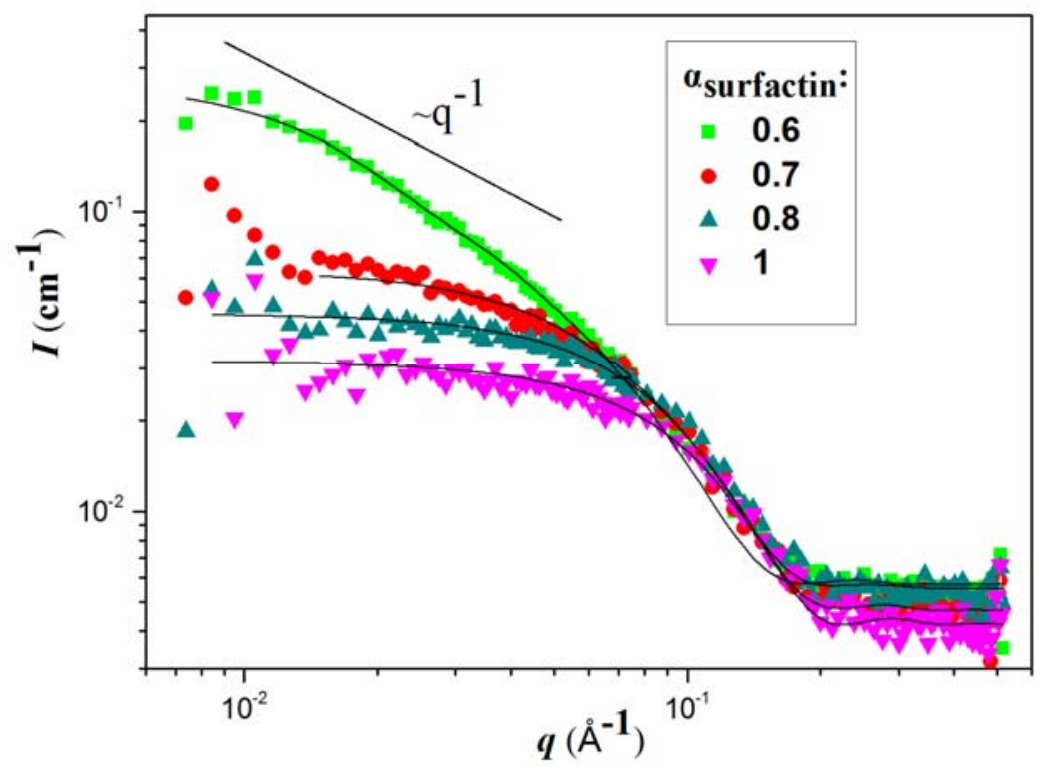

356

357

358

359

360

361

362

363

364

365

366

367

Figure5.The SANS results of Surfactin/12-3-12 mixtures at different $\alpha_{\text {Surfactin }}$ values.Solid lines are fits according to the models described in the text below.

At constant concentration the scattering intensities $I(q)$ are proportional to thevolume square of aggregates and the contrast between neutron scattering length densities of the aggregates and the solvent ${ }^{47}$. Figure 5reveals that the scattering intensity decreases with the increase of $\alpha_{\text {SurfactinValue. This result is consistent with the }}$ above results in Section 3.2 that there is synergetic interaction between Surfactin and Gemini, wherethe interaction parameters between Surfactin and 12-3-12 decreasein the same $\alpha_{\text {Surfactin }}$ range. We have performed the data analysis by employing the Indirect Fourier Transformation (IFT) method developed by Glatter ${ }^{48}$ and using the version reported by J. S. Pedersen ${ }^{49}$. 

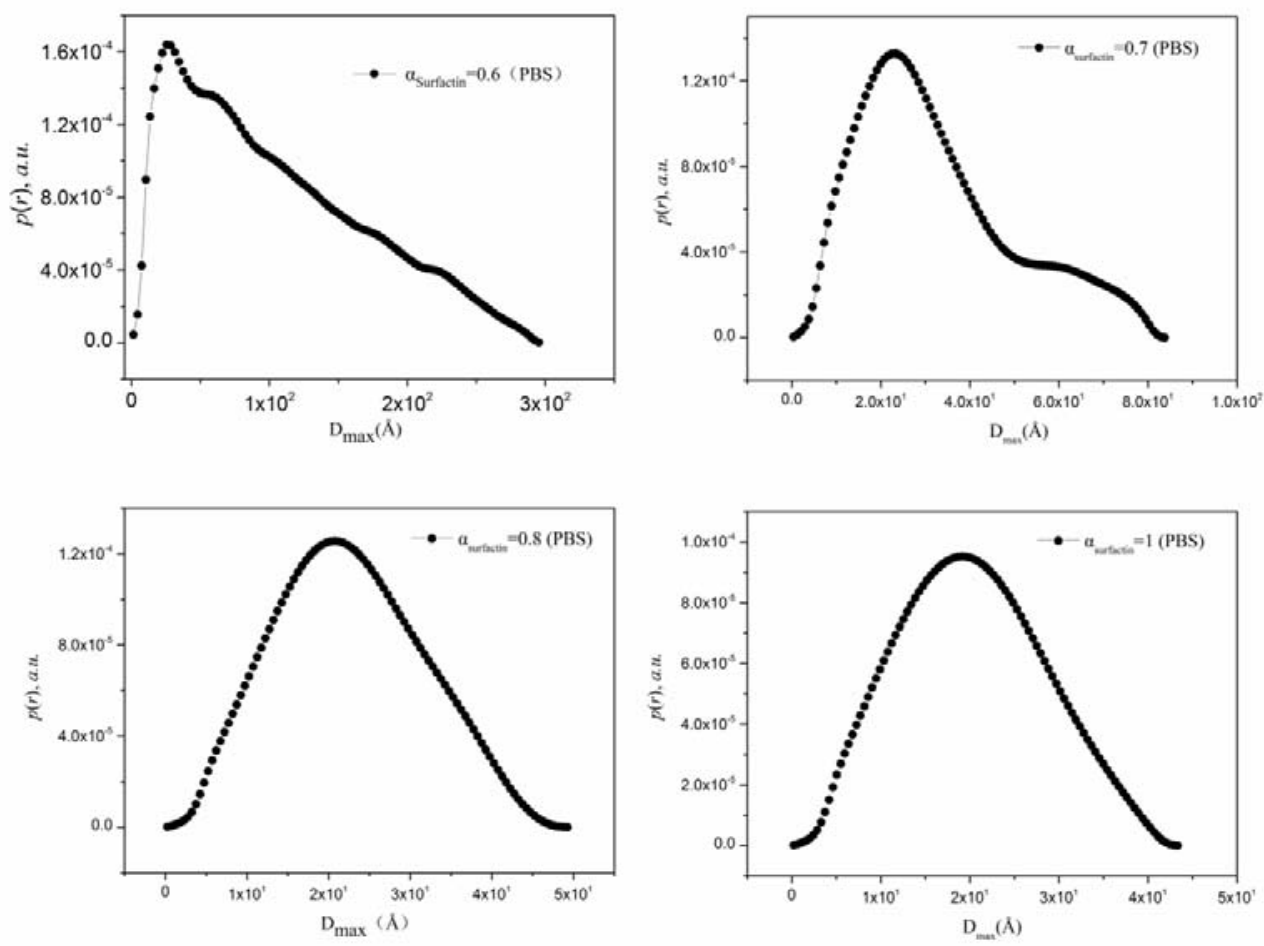

Figure 6.P(r) function obtained from the correspondingscattering curves in Figure 5.

The $p(\mathrm{r})$ functionsfor $\alpha_{\text {Surfactin }}=0.6-1$ were presented inFigure 6 . The estimation of the micelle diameter $D_{\max }$ was obtained from the maximum distance of $P(\mathrm{r})$, and all the fitted data are listed in Table 3. $p(\mathrm{r})$ function for $\alpha_{\text {Surfactin }}=0.6$ in Figure 6 reveals that largeaggregates form in the system. Table 3 shows thatradius of gyration $\mathrm{R}_{\mathrm{g}}$ for $\alpha_{\text {Surfactin }}=0.6$ is $87 \AA$, which is highestamong these systemsand with the DLS results. The slope of scattering curve around -1 and shape of $p(r)$ function of $\alpha_{\text {Surfactin }}$ $=0.6$ indicates thatunspherical (elongated) aggregates form in the solution. The overall size of aggregates is too large for measured $q$ range and it is possible to estimate that length of aggregates is larger than $300 \AA$.The data have been analysed by IFT in approximation of infinitely long cylinder.In this case the information about cross section properties as pair distance distribution function of cross section $\mathrm{p}_{\mathrm{CS}}(\mathrm{r})$ and diameter of cross section approximately $50 \AA$ have been obtained. When $\alpha_{\text {Surfactin }}=$ $0.7, p(\mathrm{r})$ function shows that both spherical micelles (symmetric part) with $D_{\max }=50$ Åand rod like micelles with length of $80 \AA$ are formed $\left(p(r)\right.$ with larger $D_{\max }$ of $100 \AA$ for $_{\text {Surfactin }}=0.7$ was also calculated and shown in supporting info ). Besides, the shape 
of $P(\mathrm{r})$ function of $\alpha_{\text {Surfactin }}=0.8$ and 1 change to symmetric shows that the aggregates shape is close to spherical.

To confirm assumption from shape of $\mathrm{p}(\mathrm{r})$ function the direct modelling has been performed ${ }^{[50]}$. At high Surfactin content the model fitting has been done with model of elliptical object with semi axis of a, b, b (model fits are shown as lines in Fig. 5) . The parameters $\mathrm{a}=\mathrm{b}=20 \pm 1 \AA$ for $\alpha_{\text {Surfactin }}=1, \mathrm{a}=20 \pm 1 \AA$ and $\mathrm{b}=26 \pm 1 \AA$ for $\alpha_{\text {Surfactin }}=0.8$ were obtained. At $\alpha_{\text {Surfactin }}=0.6$ the fit with model of cylinders with length of $280 \pm 10 \AA$ and radius of cross section of $20 \pm 1 \AA$ shows some deviation in intermediate q range which can point on presence of small amount of small spherical aggregates. For the most complicated case of $\alpha_{\text {surfactin }}=0.7$, it is possible to fit data with mixture of volume fraction 0.33 of cylinders of length $80 \pm 5 \AA$ and cross section radius of $20 \pm 1 \AA$ and volume fraction 0.67 of spherical objects of radius $20 \pm 1 \AA$. Due to limited q range and SANS data measured only at one scattering contrast the fit parameters should be consider with caution.

The shape of the surfactant aggregates in solution could bepredicted by the critical packing parameter (cpp) which is defined as cpp $=V_{\mathrm{c}} / A l_{\mathrm{c}}$, where $V_{\mathrm{c}}$ is the alkyl chain molecular volume, $l_{\mathrm{c}}$ is the extended chain length, and $A$ is the areas per molecule. The value $A$ was measured to be $0.78 \pm 0.04 \mathrm{~nm}^{2}$ for $12-3-12^{51}$ and $1.47 \pm 0.05 \mathrm{~nm}^{2}$ for Surfactin $^{52}$ by Neutron reflectometry. The variables $l_{\mathrm{c}}$ and $V_{\mathrm{c}}$ can be calculated by Tanford's formula, eqs6 and $7 . .^{53}$

$$
\begin{aligned}
l_{c} & =\left(0.154+0.127 n_{c}\right) \mathrm{nm} \\
V_{C} & \approx\left(0.0274+0.0269 n_{c}\right) \mathrm{nm}^{3}
\end{aligned}
$$

where $n_{c}$ is the number of methylene groups on thehydrocarbon chain of the surfactant. For Gemini surfactants, the hydrocarbon tail chains cannot extend beyond a certain length $l_{\mathrm{c}}$, and each tail must occupy a certain volume $V_{\mathrm{c}} \cdot{ }^{54}$ Surfactants with the cpp value of less than $1 / 3$ are supposed toform spherical micelles. When the cpp value is between $1 / 3$ and $1 / 2$, it indicates that thesurfactant is to formrod-shaped micelles, whereaswhen the cpp value is between $1 / 2$ and 1 , it generally corresponds to a monolayer or multilayer vesicles. The cpp value is $0.142 \pm 0.05$ for surfactinwhich is below $1 / 3$,suggesting thatthey form spherical micelles in solution. Whilethe value 
407 is $0.514 \pm 0.027$ for $12-3-12$ which isclose to $1 / 2$, it indicates that they formrodlike 404 micelles or vesiclesin solution.In a word, The cpp value for 12-3-12 ismuch bigger 40 than Surfactin, If the surfactant composition at interface isnonuniform, with each 47e dominating in regions of preferredcurvature, then the spherical Surfactin micelles can $47 \%$ be deformedinto rod-like micelles as $12-3-12$ is increasinglyadded to the system(see 478 in figure 7).

429 Table 3. The fitted results of SANS Data for the Surfactin and 12-3-12 Mixtures

\begin{tabular}{cccc}
\hline$\alpha_{\text {Surfactin }}$ & $D_{\max }(\AA)$ & $I(0),\left(\mathrm{cm}^{-1}\right)$ & $R_{g},(\AA)$ \\
\hline 0.6 & 300 & $0.28 \pm 0.01$ & $87 \pm 4$ \\
0.7 & 85 & $0.060 \pm 0.001$ & $26 \pm 1$ \\
0.8 & 50 & $0.038 \pm 0.001$ & $17 \pm 1$ \\
1 & 44 & $0.027 \pm 0.001$ & $16 \pm 1$ \\
\hline
\end{tabular}

450

451

$45 \bar{\Delta}$

Thus it is summarized that at low $\operatorname{Gemini}\left(\alpha_{\text {Surfactin }}=0.8-1\right)$ content there aresphericalaggregates around $44 \AA$ in diameter. Withincrease of Gemini these aggregates expand and transform to short elongated aggregates $\left(\alpha_{\text {Surfactin }}=0.7\right)$. With further increase of Gemini part of aggregates in elongated form $\left(\alpha_{\text {Surfactin }}=0.6\right)$.

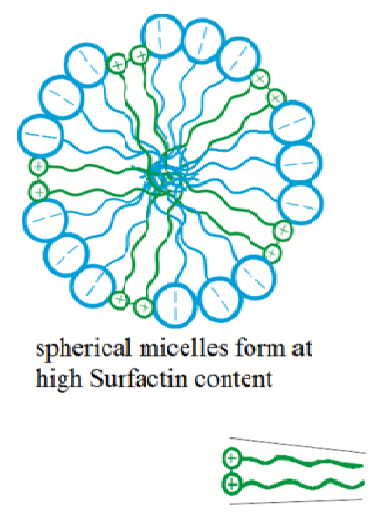

$12-3-12$

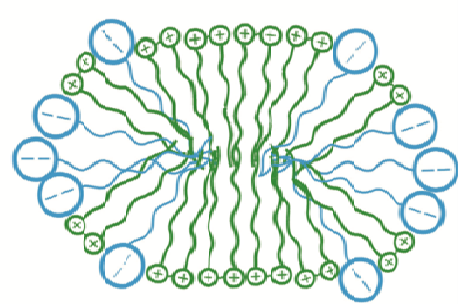

rod like micelles form at low Surfactin content



Figure 7.Sketch of the mixed surfactants micellesin the bulk solution.

\section{Conclusions}

Surfactin, a biosurfactanthas large potential applications in industrial, biotechnological, and therapeuticalfields $s^{17,18}$ due to its high surface activity and less toxic. To minimize the cost ofusing biosurfactantSurfactin, there are a number of 
studies on Surfactin/syntheticsurfactants mixturesin recent years. These synthetic surfactants include anionicSodium Dodecylbenzenesulphonate(SDOBS) ${ }^{55}$, ampholyticBetaines $^{26}$ and cationicCTAB ${ }^{28}$. However, the precipitation condition and the aggregatesproperties (shape and size)of suchSurfactin/cationic surfactants system were still unclear.

In the present work, we study the precipitation phase boundary and micellarproperties of binary systems formed by Surfactin and Cationic Gemini surfactant 12-3-12. Precipitation phase boundary of mixed Surfactin and 12-3-12 were evaluated to ensure that the surface tension measurements are in regions without precipitate. At high concentrations, the solutions are turbid, but with the increasing molar fraction of Surfactin $\left(\alpha_{\text {Surfactin }}\right)$ turbidity weakened. The CMC analysis shows that the mixtures exhibit synergism in the formation of Surfactin/12-3-12 mixed micelles. DLS and SANS results indicated that in the high 12-3-12 content region larger aggregates were formed, and with the increasing of $\alpha_{\text {Surfactin, }}$ micelles of smaller size were formed, so the system became more stable. Besides elongated micelles are formed at $\alpha_{\text {Surfactin }}=0.6$, mixture of elongated and spherical micelles at $\alpha_{\text {Surfactin }}=0.7$ and only spherical micelles at $\alpha_{\text {Surfactin }}=0.8,1$.

It is interesting to note that Surfactin may have the capability to reduce precipitation when mixed with cationic surfactants due to the peptide ring. The results of Wang ${ }^{45}$ also support the conjecture which has been discussed in section 3.2. In addition, the asymmetrically double-tailed cationic surfactants ${ }^{10}$ and extended anionic surfactants ${ }^{35}$ have been reported to have the similar property to minimize precipitation when mixed with opposite-charged surfactants. It is worth mentioning thatSurfactin/12-3-12 mixtures can reduce the interfacial tension to an ultralow level in alkaline environment(see details in Supporting Information). These fundamental physicochemical properties are favorable to the use of Surfactin/Gemini mixture in daily chemical and oil chemistry field.

\section{Supporting Information}

The experimental details on the dynamic interfacial tension (IFT) between surfactin/12-3-12 and crude oil in alkaline solution. The 1H NMR Spectra and the 
related analyzing date of 12-3-12. Details on the new approach to describe the thermodynamic stability of mixed micelle using Gibbs energy of micellization.p(r) with larger $D_{\max }$ of $100 \AA$ for $\alpha_{\text {Surfactin }}=0.7$.

\section{Acknowledgements}

We gratefully acknowledge the support of this work by Shanghai Natural Science Foundation (Grant No. 15ZR1409900), the National Natural Science Foundation of China (No. 21573070), and Fundamental Research Funds for the Central Universities. This work benefited from the use of the SasView application, originallydeveloped under NSF award DMR-0520547.

\section{References:}

(1) Y.Konodo, H.Uchiyama, N.Yoshino, K.Nishiyama, M.Abe, Spontaneous Vesicle Formation from Aqueous Solutions of Didodecyldimethylammonium Bromide and Sodium Dodecyl sulfate Mixtures, Langmuir., 11 (1995)2380-2384.

(2) A.Khan, E.F.Marques, Synergism and Polymorphism in Mixed Surfactant Systems,Curr.Opin.ColloidInterf. Sci. 4 (1999)402-410.

(3) C.Tondre, C.Caillet, Properties of the Amphiphilic Films in Mixed Cationic/Anionic Vesicles: A Comprehensive View from a Literature Analysis,Adv. Colloid Interf. Sci. 93 (2001)115-134.

(4) J.M.Peacock,E.Matijevic,Precipitation of alkylbenzenesulfonates with metal ions, J Colloid Interface Sci.77 (1980)548-554.

(5) N.Kallay,M.Pastuovic,E.Matijevic, Solubility and enthalpy of precipitation of magnesium, calcium, strontium, and barium dodecyl sulfates, J Colloids Interface Sci. 106 (1985)452-458.

(6) A. Mehreteab,F.J.Loprest, Formation of pseudo-nonionic complexes of anionic and cationic surfactants, J Colloid Interface Sci. 125(1988)602-609.

(7)Z.J. Yu,G.Xu, Physicochemical properties of aqueous mixtures of tetrabutylammonium bromide and anionic surfactants. 1. Temperature-induced micellar growth and cloud point phenomenon,J PhysChem.93(1989)7441-7445.

(8) Z.J. Yu,Z. Zhou, G.Xu, Physicochemical properties of aqueous mixtures of tetrabutylammonium bromide and anionic surfactants. 2. Micellar growth patterns and 
the effect of intermicellar interactions on light-scattering data,J Phys. Chem. 93 (1989) 7446-7451.

(9) Z.J. Yu, X. Zhang,G. Xu,G.X.Zhao, Physicochemical properties of aqueous mixtures of tetrabutylammonium bromide and anionic surfactants. 3. Effects of surfactant chain length and salinity,J PhysChem.94 (1990) 3675-3681. (10)A.Upadhyayaa,E.J.Acostab,J.F.Scamehornc,D.A. Sabatinid,Microemulsion Phase Behavior of Anionic-Cationic Surfactant Mixtures: Effect of Tail Branching,J SurfactDeterg. 9(2006), 169-179.

(11)M. Bergstrom,J.S. Pedersen, Formation of tablet-shaped and ribbonlike micelles in mixtures of an anionic and a cationic surfactant,Langmuir. 15 (1999) 2250-2253. (12)M. Bergstrom,J.S. Pedersen,P. Schurtenberger,S.U.Egelhaaf, Small-angle neutron scattering (SANS) study of vesicles and lamellar sheets formed from mixtures of an anionic and a cationic surfactant,J PhysChem B.103 (1999) 9888-9897.

(13) M. Bergstrom, J.S.Pedersen, A small-angle neutron scattering study of surfactant aggregates formed in aqueous mixtures of sodium dodecyl sulfate and didodecyldimethylammonium bromide,J PhysChem B.104 (2000) 4155-4163.

(14) F.Baumgart,B.Kluge, C.Ullrich,J.Vater, D.Ziessow, Identification of amino acid substitutions in the lipopeptideSurfactin using 2D NMR spectroscopy. Biochem.Biophiys, Res. Commun.177(1991)998-1005.

K.Oka,T.Hirano,M.Homma,H.Ishii,K.Murakami,S.Mogami,A.Motizuki,H.Morita,K.T akeya,H.Itokawa, Satisfactory separation and MS-MS spectrometry of six Surfactins isolated from Bacillus subtilisnatto,Chem. Pharm. Bull. 41(1993) 1000-1002.

(16) N.Hue,L.Serani,O.Laprevote, Structural investigation of cyclic peptidolipids from Bacillus subtilis by high-energy tandem mass spectrometry, Rapid Commun. Mass Spectrom.15 (2001) 203-209.

(17) A.H.Zou,J.Liu,V.M.Garamus,K.Zheng,R.Willumeit,B.Z. Mu, Interaction between the Natural Lipopeptide [Glu1, Asp5] Surfactin-C15 and Hemoglobin in Aqueous Solution, Biomacromolecules. 11(2010)593-599.

(18) C.N.Mulligan, Environmental applications for biosurfactants,Env.Pol. 133 (2005) 
183-198.

(19) K.Ma, E.L.Clancy, Y.Zhang, D.G.Ray, K.Wollenberg, M.G.Zagorski, Residue-Specific pKa Measurements of the $\beta$-Peptide and Mechanism of $\mathrm{pH}$-Induced Amyloid Formation,J. Am. Chem. Soc. 121 (1999) 8698-8706.

(20) F.M.Menger, C.A.Littau, Activation of hydrocarbons by a ruthenium(II) (fluoroalkyl)phosphine hydride complex, J. Am. Chem. Soc. 113 (1991) 1450-1451. (21) F.M.Menger,C.A.Littau, Gemini surfactants: a new class of self-assembling molecules,J. Am. Chem. Soc. 115(1993)10083-10090.

(22) R.Zana,Dimeric and oligomeric surfactants. Behavior at interfaces and in aqueous solution: a review,Adv Colloid and Interface Sci.97(2002) 205-253.

(23)J. Zhao, D.C. Sherril, B.M. Fung, Mixtures of Monomeric and Dimeric Cationic Surfactants,J. Phys. Chem. B.102 (1998) 7613-7618.

(24)D.Danino,Y.Talmon,R.Zana,Alkanediyl- $\alpha, \omega$-Bis(Dimethylalkylammonium

Bromide) Surfactants (Dimeric Surfactants). 5. Aggregation and Microstructure in Aqueous Solutions,Langmuir. 11 (199) 1448-1456.

(25) A.H.Zou,J.Liu,V.M.Garamus, Y.Yang,R. Willumeit,B.Z.Mu,Micellization Activity of the Natural Lipopeptide [Glu1, Asp5] Surfactin-C15 in Aqueous Solution, J. Phys. Chem. B. 114 (2010) 2712-2718.

(26) F.Liu,J.W.Xiao,V.M.Garamus,L.Almasy,R.Willumeit,B.Z.Mu,A.H. Zou, Interaction of the Biosurfactant, Surfactin with Betaines in Aqueous Solution,Langmuir. 29(2013)10648-10657.

(27) J.W.Xiao,F.Liu, V. M.Garamus,L.Almásy, U.A.Handge,R.Willumeit,B. Z.Mu, A.H.Zou, Insights into the Interactions among Surfactin, Betaines, and PAM:Surface Tension, Small-Angle Neutron Scattering, and Small-Angle $\mathrm{X} \square$ ray Scattering Study,Langmuir. 30 (2014) 3363-3372.

(28)H. Wang, H.Z. Gang, R.Q. Ye, B.Z. Mu, Interaction between biosurfactantsurfactin and cationicsurfactant cetyltrimethyl ammonium bromide in mixed micelle, Colloid Polym Sci.292 (2014) 3169-3176.

(29)Y.M.Li, N.A.Haddad, S.Z.Yang, B.Z.Mu, Variants of Lipopeptides Produced by Bacillus licheniformis HSN221 in Different Medium Components Evaluated by a 
550 Rapid Method ESI-MS， Int. J. Pept. Res. Ther. 14 (2008) 229-235.

551 (30) C.S.Song, R.Q.Ye, B.Z.Mu, Molecular Behavior of a Microbial Lipopeptide

552 Monolayer at the Air-Water Interface, Colloid Surf. A. 302 (2007), 82-87.

553 (31) R.Zana, M.Benrraou, R.Rueff, Alkanediyl- $\alpha, \omega$-bis(dimethylalkylammonium

554 bromide) Surfactants. 1. Effect of the spacer chain length on the critical micelle

555 concentration and micelle ionization degree,Langmuir.7(1991) 1072-1075.

556 (32)L.A.Bastardo,V.M.Garamus, M.Bergström, P.M.Claesson, The Structures of

557 Complexes between Polyethylene Imine and Sodium Dodecyl Sulfate in D2O: A

558 Scattering Study,J. Phys. Chem. B. 109 (2005) 167-174.

559 (33) A.Krezel, W. Bal, A Formula for CorrelatingpKa Values Determined in $\mathrm{D}_{2} \mathrm{O}$ and

$560 \quad \mathrm{H}_{2} \mathrm{O}$,J. Inorg. Biochem. 98 (2004) 161-166.

561 (34) X.J.Fan,P.Stenius,N.Kallay,E.Matijevic,Precipitation of Surfactant Salts II. The

562 Effect of Nonionic Surfactants on Precipitation of Calcium Dodecyl Sulfate, J

563 COLLOID INTERF SCI.,121 (1988) 571-578.

564 (35) D.Panswad,D.A.Sabatini, S.Khaodhiar,Precipitation and Micellar Properties of

565 Novel Mixed AnionicExtended Surfactants and a Cationic Surfactant,J

566 SurfactDeterg. 14 (2011) 577-583.

567 (36)A. Heinemann,S.Mühlbauer,SANS-1: Small angle neutron scattering,Journal of 568 large-scale research facilities.http://dx.doi.org/10.17815/j1srf-1-32, 2015(accessed 569 16.03.22).

570 (37) Pipich,V.QtiKWS: user-friendly program for reduction, visualization, analysis 571 and fit of SA(N)S data.URL http://www.qtikws.de, 2007(accessed 16.03.22).

572 (38) R.Zana, Alkanediyl- $\alpha, \omega$-bis(dimethylalkylammonium bromide) Surfactants:II.

573 Krafft Temperature and Melting Temperature,Journal of Colloid and Interface 574 Science.252 (2002) 259-261.

575 (39) J.M.Bonmatin, M.Genest, H.Labbe,M.Ptak, Solution three-dimensional structure 576 of surfactin: A cyclic lipopeptide studied by $1 \mathrm{H}-\mathrm{nmr}$, distance geometry, and 577 molecular dynamics, Biopolymers. 34 (1994) 975-986.

578 (40) J.F.Scamehorn,J.H.Harwell,Surfactant-Based Separations. Science and 579 Technology, American Chemical Society, New York, 2005, pp.601. 
(41) J.H.Harwell, Factors Affecting Surfactant Performance in Groundwater Remediation Applications, in Transport and Remediations of Subsurface Contaminants,ACS, Washington DC, 1992, pp. 124.

(42) Y.Z.Shang,T.F.Wang, X.Han,C.J.Peng,H.L.Liu, Effect of Ionic Liquids $\mathrm{C}_{\mathrm{n}} \mathrm{mimBr}$ on Properties of Gemini Surfactant 12-3-12 Aqueous Solution,Ind. Eng. Chem. Res. 49 (2010) 8852-8857.

(43)S. Chavda,P. Bahadur,V.K.Aswal,Interaction Between Nonionic and Gemini (Cationic) Surfactants: Effect of Spacer Chain Length,JSurfactDeterg. 14 (2011) $353-362$.

(44) D.N.Rubingh, Mixed Micelle Solutions. In Solution Chemistry of Surfactants. Springer, New York, 1979,pp.337-354.

(45)Rosen, M.J.Surfactants and interfacial phenomena,3rd edn.Wiley, New Jersey, 2004,pp. 386-396.

(46) H.Maeda, Simple thermodynamic analysis of stability ofionic/nonionic mixed micelles,J Colloid Interface Sci.172(1995) 98-105.

(47)X.M. Yang,L. Zhao,L. Almasy,V.M.Garamus,A.HZou, R. Willumeit, S.J. Fan,International Journal of Pharmaceutics.450 (2013) 225- 234.

(48)O.Glatter, A New Method for the Evaluation of Small-AngleScattering Data,J. Appl. Crystallogr. 10 (1977) 415-421.

(49)J.S.Pedersen, Analysis of Small-Angle Scattering Data from Colloids and Polymer Solutions: Modeling and Least-Squares Fitting,Adv.ColloidInterf. Sci.70 (1997) 171-210.

(50) I.Breßler, J.Kohlbrecher, A,F.Thünemann. SASfit : a tool for small-angle scattering data analysis using a library of analytical expressions. Journal of Applied Crystallography, 48(2015):1587-1598.

(51) H.H Shen, R.K. Thomas, C.Y.Chen, Aggregation of the Naturally Occurring Lipopeptide, Surfactin, at Interfaces and in Solution: An Unusual Type of Surfactant, Langmuir, 25(2009)4211-4218.

(52) X.L.Pei, C.D. Chu, R.K. Thomas, Adsorption of Gemini Surfactants with Dodecyl Side Chains and Different Spacers, Including Partially Fluorinated Spacers, 


610 on Different Surfaces: Neutron Reflectometry Results, Langmuir,
611 27(2011):1844-1852.
612 (53)C.Tanford, The Hydrophobic Effect: Formation of Micelles andBiological
613 Membranes, Wiley, New York, 1980, pp. 51-53.
614 (54)R. Zana, GEMINI SURFACTANTSSynthesis, Interfacial and Solution-Phase
615 Behavior, and Applicalions,MARCEL DEKKER, INC: New York,2004,pp. 41-42.
616 (55) S.A Onaizi, MS Nasser, F.A Twaiq,Micellization and interfacial behavior of a
617 synthetic surfactant-biosurfactant mixture, Colloids \& Surfaces A,
618 415(2012):388-393.




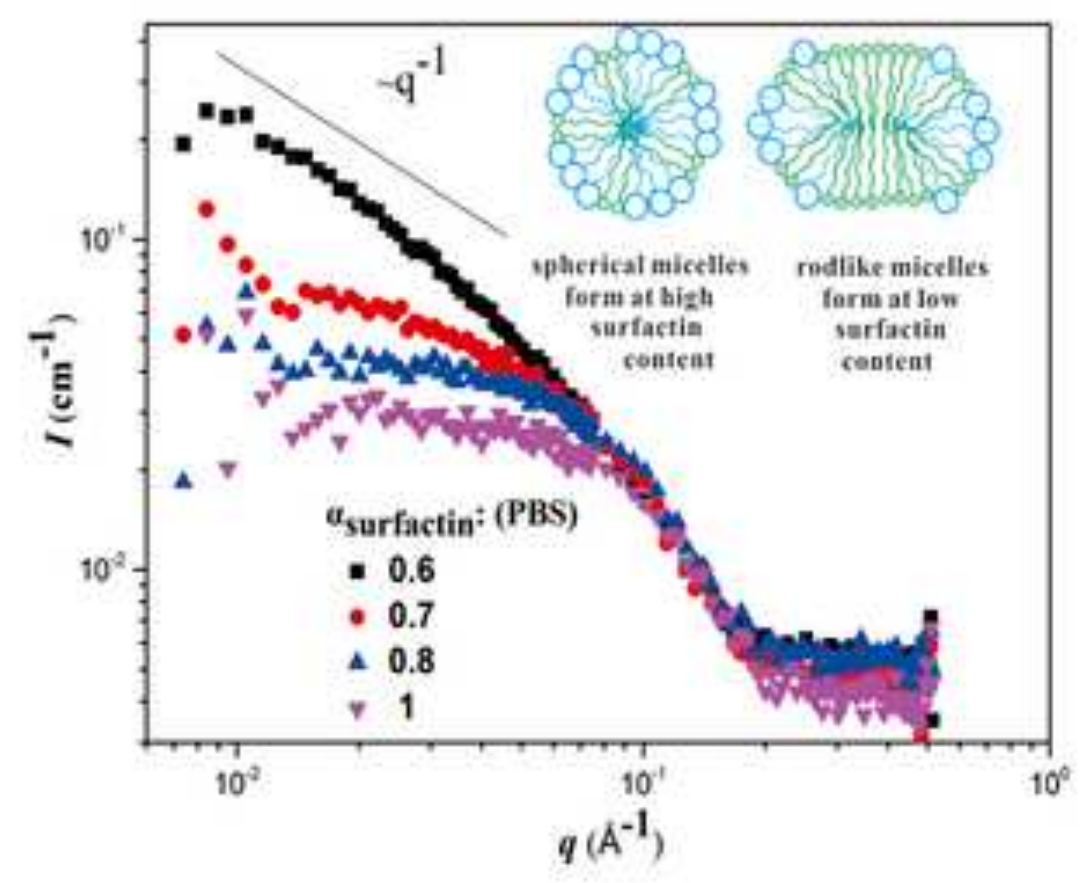

